\title{
Micro Computed Tomography Analysis of Four-Way Conversion Catalysts using Artificial Intelligence-Enabled Image Processing
}

\author{
Robert Palomino ${ }^{1}$, Ke-Bin Low ${ }^{1}$, Chunxin $\mathrm{Ji}^{2}$, Ivan Petrovic ${ }^{2}$, Florian Waltz ${ }^{3}$ and Thomas $\mathrm{Schmitz}^{3}$ \\ ${ }^{1}$ BASF Corporation, Iselin, New Jersey, United States, ${ }^{2}$ BASF Corporation, United States, ${ }^{3}$ BASF Catalysts \\ Germany GmbH, United States
}

\section{Introduction}

With stricter particulate matter (PM) emission regulations, PM filters coated with a three-way conversion catalyst (TWC), named four-way catalysts $\left(\mathrm{FWC}^{\mathrm{TM}}\right)$, are being deployed in gasoline-engine powered vehicles. Most FWCs consist of TWC formulations coated either on-walls or in-walls of high-porosity ceramic filter substrates. Typical visualization method for characterizing these catalyzed filters is the scanning electron microscope (SEM), which also can overlay elemental distribution information using Energy-Dispersive X-Ray Spectroscopy (EDS).

However, SEM/EDS requires samples to be cut, mounted and polished for cross-sectional analysis, which only offers limited two-dimensional (2D) views from specific depth of bulk filters. Moreover, complete pore network openness and wash coat deposition profiles are not detectable using 2D imaging. This type of 3D visualization and analysis is critical in FWC development, which are directly related to filter back-pressures and catalytic activities [1].

Laboratory-based Micro Computed Tomography $(\mu \mathrm{CT})$, although limited in spatial resolution, is able to bridge the gap by offering three-dimensional (3D) visualization and a significantly larger field-of-view. Tomograms from $\mu \mathrm{CT}$ commonly relies on differences in X-ray absorption or phase properties to generate contrasts. For FWCs, $\mu$ CT contrast between wash coat and substrate can be subtle, and signal-to-noise is usually weak as we push towards the instrument's resolution limit. In order to elucidate filter pore network and wash coat deposition profiles, we apply artificial intelligence (AI)-driven computer vision models, trained via deeplearning, on $\mu \mathrm{CT}$ datasets to denoise and classify features at the pixel-level for semantic segmentation. The segmented tomograms enable quantitative analysis in 3D that offers insight into structure-property relationships for FWCs.

\section{Materials and Methods}

Cordierite based filter substrates were coated with identical TWC catalyst slurries and at the same nominal wash coat loading in term of $\mathrm{g} / \mathrm{in}^{3}$. But two FWC samples showed different back-pressures using two different coating parameter sets. They were cored and trimmed down to approximate dimensions of $4 \times 4 \times 44 \mathrm{~mm}$. The $\mu \mathrm{CT}$ acquisitions were performed using a Zeiss Xradia 520 Versa X-Ray microscope with $160 \mathrm{kV}$ accelerating voltage and $10 \mathrm{~W}$ power. The tomograms were then exported into 2000 two-dimensional slices with pixel resolution of about $2.3 \mu \mathrm{m} / \mathrm{px}$, of which four to six were used for deep-learning to build semantic segmentation models. Training images were labeled based on four feature classes: Wash coat, Substrate, Pores and Open Channel; and subsequently fed into MIPAR Image Analysis deep-learning module [2]. Quantitative analysis and visualization of the segmentation in 3D were done using a combination of MIPAR Image Analysis and ImageJ [3].

\section{Results and Discussion}

Semantic segmentation using the trained models successfully separated the wash coat from the cordierite substrate. The calculated porosity of cordierite substrate agrees very well with the experimentally measured 
values. Vastly different wash coat distributions on and across the filter walls were revealed as well, which otherwise cannot be observed easily from the raw tomograms (Figure 1a). For the FWC sample coated by parameter set-A, most of the wash coat was deposited into the porous walls, resulting in a significant fraction of pores that are open to the channels (Figure 1b). In contrast, the FWC sample prepared by parameter set-B yielded over $30 \%$ increase in wash coat volume deposited on the filter walls compared to parameter set-A, hence blocking most of the surface pores, causing undesirable high backpressure (Figure 1c). Statistical analysis of wash coat deposition locations and profiles within the filter walls provided more insights about catalyst slurry penetration and settling processes, which will guide future FWC improvements.

\section{Significance}

Artificial intelligence-driven computer vision enables robust semantic segmentation for tomographic data that enhances 3D structural elucidation and paves the way for quantitative analysis.
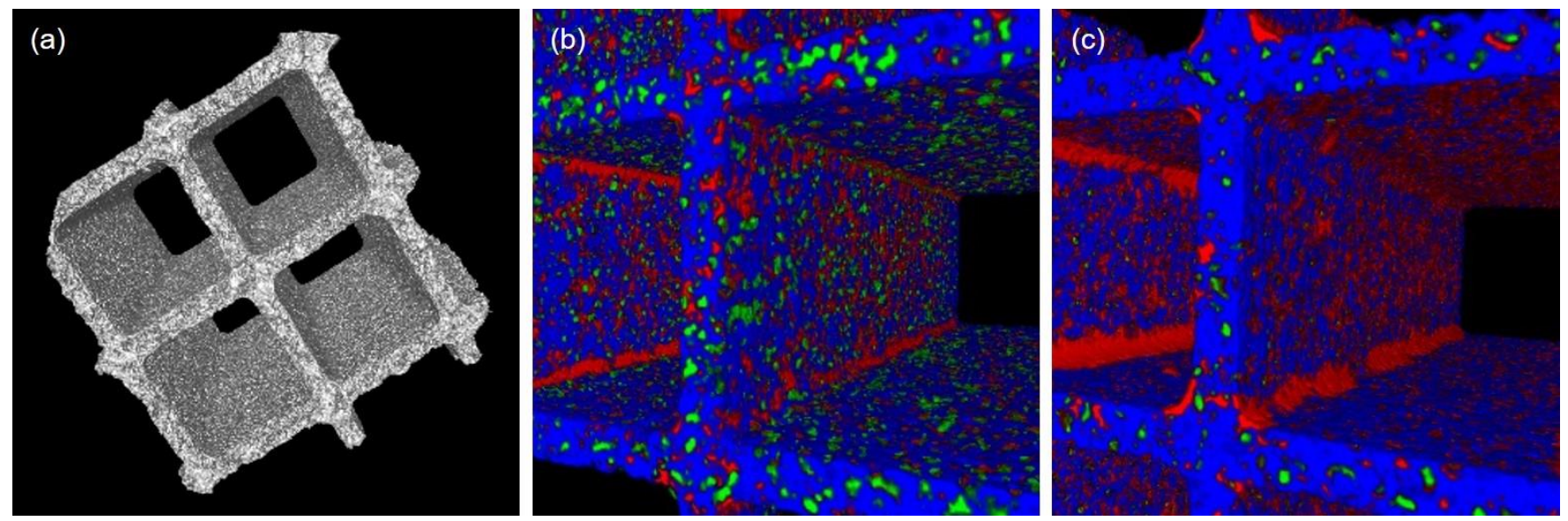

Figure 1. (a) Raw $\mu \mathrm{CT}$ tomogram of FWC; Close-up view of segmented FWC coated by (b) parameter setA, and (c) parameter set-B. Red denotes Washcoat; Blue denotes Substrate; Green denotes Unfilled Pores.

\section{References}

1. Belot, I, Vidal D., Greiner R., Votsmeier, M., Hayes, R. E., Bertrand. F., Chemical Engineering Journal, in press (2020).

2. Sosa, J.M., Huber, D.E., Welk, B., Fraser, H.L., Integrating Materials and Manufacturing Innovation 3, 123-140 (2014).

3. Schindelin, J., Arganda-Carreras, I., Frise, E., Kaynig, V., Longair, M., Pietzsch, T., Preibisch, S., Rueden, C., Saalfeld, S., Schmid, B., Tinevez, J., White, D.J., Hartenstein, V., Eliceiri, K., Tomancak, P., Cardona, A., Nat Methods 9, 676-682 (2012). 